\title{
CARACTERÍSTICAS QUE SE ASOCIAN CON LA OMISIÓN DEL DESAYUNO EN ADOLESCENTES MONTEVIDEANOS QUE CONCURREN A COLEGIOS PRIVADOS
}

\author{
CHARACTERISTICS ASSOCIATED WITH THE OMISSION OF BREAKFAST IN MONTEVIDEAN ADOLESCENTS \\ CONCURRING TO PRIVATE SCHOOLS
}

CARACTERÍSTICAS QUE SE ASSOCIAM COM A OMISSÃO DO CAFÉ DA MANHÃ EM ADOLESCENTES MONTEVIDEANOS QUE COINCIDEM A COLÉGIOS PRIVADOS

\section{Florencia Ceriani- Infantozzi}

Universidad de la República. Montevideo, Uruguay.

florceriani@gmail.com

ORCID: 0000-0003-2105-8044

\section{Carolina De León- Giordano}

Universidad Católica del Uruguay. Montevideo, Uruguay.

Red de Uruguaya de Apoyo a la Nutrición y Desarrollo Infantil. Uruguay.

DOI: https://doi.org/10.22235/ech.v6i2.1461

ORCID: 0000-0001-6777-3946

Recibido el 23/02/2017

Aceptado el $07 / 08 / 2017$

\section{RESUMEN}

El objetivo del artículo es conocer las características que se asocian a la omisión del desayuno de adolescentes de 11 a 17 años que asisten a colegios privados de Montevideo. Estudio transversal, descriptivo, analítico mediante el uso de una base de datos secundaria. Se estudió la asociación entre la omisión del desayuno y diferentes variables, con 696 estudiantes y luego con la submuestra de 196 que omiten desayunar. Se calculó Test t de Student, se aplicó el test chi cuadrado de Pearson. Para identificar el riesgo se calculó el OR. Valor significancia estadística $\mathrm{p}<0,05$. Procesamiento y análisis estadístico SPSS versión 21.0. El 28.2\% de los adolescentes omitió desayunar. La probabilidad de omitir el desayuno con respecto a no omitirlo es aproximadamente 2,25 más alto en los adolescentes que se encuentran solos con respecto a los que se encuentran acompañados. Entre quienes omitían el desayuno un $40.4 \%$ presentó sobrepeso u obesidad, siendo mayor esta cifra en los que tenían 13 años o menos $(p=0.002)$. El motivo más mencionado para omitir el desayuno fue "levantarse tarde y no tener tiempo". La edad y el sexo se asociaron significativamente con el motivo mencionado. La omisión del desayuno es un problema en esta población. El sobrepeso y obesidad es una complicación de gran magnitud entre quienes omiten desayunar. Estar sólo en este tiempo de comida aumenta las chances de omitirlo.

Palabras clave: Desayuno, Conducta Alimentaria, Nutrición del Adolescente, Dieta.

\section{ABSTRACT}

The objective of the article is to know the characteristics that are associated to the omission of the breakfast of adolescents of 11 to 17 years who attend private schools of Montevideo. Cross-sectional, descriptive, analytical study using a secondary database. We studied the association between breakfast omission and different variables, with 696 students and then with the subsample of 196 that skipped breakfast. Student's $t$ test was calculated, Pearson's chi square test was applied. To identify the risk, the OR was calculated. Statistical significance value $p<0.05$. Processing and statistical analysis SPSS version 21.0. $28.2 \%$ of adolescents skipped breakfast. The probability of omitting breakfast with respect to not omitting it is approximately 2.25 higher in adolescents who are alone with respect to those who are accompanied. Among those who skipped breakfast, $40.4 \%$ were overweight or obese, with a higher number in those aged 13 years or younger $(p=0.002)$. The most mentioned reason for omitting breakfast was "getting up late and not having time". Age and sex were significantly associated with the reason mentioned. The omission of breakfast is a problem in this population. Being overweight and obese is a major complication among those who skip breakfast. Being alone in this time of food increases the chances of omitting it.

Key words: Breakfast, Feeding Behavior, Adolescent Nutrition, Diet. 


\section{RESUMO}

O objetivo deste artigo é conhecer as características associadas à omissão de adolescentes de pequeno-almoço com idade entre 11 e 17 anos que frequentam escolas particulares em Montevidéu. cruzar estudo analítico descritiva usando um banco de dados secundário. a associação entre a saltar o pequeno almoço e variáveis diferentes, com 696 alunos e depois com a subamostra de 196 que o desjejum foram estudados. 0 teste $\mathrm{t}$ de Student foi calculado, o teste foi aplicado Pearson qui-quadrado. Para identificar o risco foi calculado o OR. Valor de significância estatística de $p<0,05$. Processamento e análise estatística SPSS versão $21,0.28,2 \%$ dos adolescentes desjejum. A probabilidade de saltar o pequeno almoço sobre não omitir é cerca de 2,25 maior nos adolescentes que estão sozinhos em relação aos quais são acompanhados. Entre aqueles omitido pequeno-almoço $40,4 \%$ tinham excesso de peso ou obesos, sendo este valor mais elevado em pacientes com 13 anos ou menos $(p=0,002)$. A razão mais citada para saltar o pequeno almoço foi "ficar até tarde e não tenho tempo." Idade e sexo foram significativamente associados com a razão mencionada. A omissão de pequeno-almoço é um problema nesta população. Sobrepeso e obesidade é uma complicação de grande magnitude entre aqueles que o desjejum. Estar sozinho neste tempo de alimentos aumenta as chances de pular.

Palavras-chave: Desjejum, Comportamento Alimentar, Nutrição do Adolescente, Dieta

\section{INTRODUCCIÓN}

La adolescencia es un período crítico de la vida, se caracteriza por un aumento en la velocidad de crecimiento y maduración, con gran desarrollo de estructuras y órganos corporales, en especial los influenciados por hormonas sexuales (1).En este momento de la vida también se establece la maduración psicológica, se instauran los hábitos dietéticos, constituyendo la base del comportamiento alimentario para el resto de la vida (2). Pareciera ser un momento clave para fomentar el hábito de desayunar.

El desayuno es considerado como una de las ingestas alimentarias más importantes del día, tal es así que la Asociación Española de Pediatría recomienda que se debe desayunar todos los días (3).Los adolescentes que realizan este tiempo de comida son capaces de afrontar mejor las actividades cognoscitivas y físicas de la mañana (4) (5). Los jóvenes que lo omiten no logran cubrir ciertos nutrientes como el calcio, potasio, fósforo, vitaminas A, C, E B6 y B12, hierro, cinc, en los siguientes tiempos de comida (6). Por otro lado, los adolescentes que no desayunan tienden a ingerir más volumen de alimentos de alta densidad calórica, con elevado aporte de grasa y azúcar refinado en el siguiente tiempo de comida, asociándose con un incremento del IMC, aumento de la circunferencia de cintura, hipertensión arterial y diabetes tipo 2 (4).
Sin embargo, a pesar de los múltiples beneficios de desayunar, es el tiempo de comida que menos interés despierta y el que más suelen omitir los jóvenes (6) (7). Múltiples son los motivos por los cuales los adolescentes suelen no desayunar (8) (9).

Según la evidencia, es más frecuente la omisión del desayuno en el sexo femenino, quizás por su mayor preocupación por su peso corporal (6) (7) (10). Con respecto a la edad, la proporción de jóvenes que realizan este tiempo de comida, desciende significativamente a medida que avanza la edad (7) (10). Otro de los factores que inciden en el hábito de desayunar es el peso corporal. Según la II Encuesta Mundial de Salud Adolescente, en los jóvenes con sobrepeso el hábito de desayunar a diario o con gran frecuencia se presenta en una menor proporción respecto a los adolescentes con estado nutricional normal, acentuándose según condición de obesidad (11).

Amat Huerta y cols. plantean que la compañía de los padres al momento de desayunar resulta ser un factor de gran relevancia (12). Se ha demostrado que los adolescentes desayunan más frecuentemente si realizan este tiempo de comida en compañía de los padres (11).

Debido a los motivos antes expresados, es que el siguiente estudio se plantea conocer las características que se asocian a la omisión del desayuno de adolescentes de 11 a 17 años que asisten a colegios privados de Montevideo.

\section{METODOLOGÍA}

Se trata de un estudio transversal, descriptivo, con componente analítico. Se trabajó con datos del estudio "Hábitos alimentarios en el desayuno de adolescentes de liceos privados de Montevideo", realizado por la Asociación Uruguaya de Dietistas y Nutricionistas (AUDYN) en el año 2012 (8). Se solicitó la correspondiente autorización por parte de AUDYN para el uso de la base de datos.

El diseño de la muestra de 696 adolescentes, realizado en el estudio de AUDYN, antes mencionado, fue aleatorio estratificado. Los estratos se constituyeron según el año que cursan los jóvenes a estudiar (primero, segundo y tercer año del ciclo básico y cuarto año o primer año de bachillerato). La unidad primaria de muestreo por estrato fueron los colegios privados habilitados de la ciudad de Montevideo y luego dentro de cada centro educativo se procedió a la selección de un grupo por cada grado escolar. Dentro de cada grupo se realizó un censo a todos los alumnos presentes que presentaron la autorización correspondiente. Se les aplicó el formulario de encuesta y se realizó la antropometría.

El formulario de encuesta fue de tipo auto-administrado, previamente validado y se realizó la medición del peso y talla con instrumentos debidamente calibrados y a cargo de licenciados en nutrición, previamente capacitados, encargados del trabajo 
de campo. Las medidas antropométricas se registraron mediante balanzas y tallímetros portátiles marca SECA. La toma de medidas fue de forma individual, en privado, y los adolescentes se presentaron con ropa liviana y sin calzado.

Se preservó el anonimato de los sujetos investigados, a cada estudiante se le asignó un código o número. Así mismo, se solicitó la firma del consentimiento informado por parte de los padres.

Para estudiar la asociación entre la omisión del desayuno y el sexo, edad, estado nutricional, la condición de estar solo durante el desayuno y la información sobre el desayuno se incluyeron todos los adolescentes de la muestra $(n=696)$. Para este análisis, la variable dependiente estudiada fue la omisión del desayuno. Esta variable fue analizada como nominal categórica (Omite el desayuno/ No omite el desayuno).

Con el fin de determinar el perfil de los adolescentes que omiten el desayuno se seleccionaron del total de la muestra de estudio aquellos adolescentes que no realizan el desayuno de forma diaria, es decir los que omiten el desayuno siempre o algunas veces, estando la submuestra de estudio conformada por 196 adolescentes.

Si bien la bibliografía sugiere la división de la edad en dos categorías: 10 a 14 años y 1519 años, para este estudio, se determinó, una clasificación de edad teniendo en cuenta que eran jóvenes de entre 11 y 17 años cuya media de edad era de 13.2 años. Se establecieron 2 categorías Menor o igual a 13 años/ Mayor de 13 años.

La variable estado nutricional, se clasifico según puntaje $Z$ de IMC/edad utilizando los estándares de la OMS (2007), para niños y adolescentes de 5 a 19 años, al igual que con el indicador Talle/edad (13).

Para determinar algunas de las características del perfil de los adolescentes que omiten el desayuno se utilizaron medidas descriptivas como media, mediana, desvíos estándar y frecuencias tanto absolutas como relativas.

Para el análisis de los motivos expresados por los adolescentes para omitir el desayuno, se utilizaron frecuencias absolutas y relativas

El análisis de la asociación entre las variables nominales categóricas estudiadas se realizó mediante la prueba de Chi cuadrado de Pearson (Chi2)

Con el fin de conocer las chances de omitir el desayuno se calculó el Odds Ratio (OR)

En todas las pruebas realizadas, se consideró significancia estadística con el valor $\mathrm{p}$ menor que 0,05.

Los datos antropométricos se procesaron con el programa Anthro Plus de la OMS (V 1.0.4). El procesamiento de datos fue realizado utilizando el paquete estadístico para Windows SPSS versión 21.0.

\section{RESULTADOS}

En relación al hábito de desayunar de esta población, los resultados muestran que un $71.8 \%$ refirió desayunar todos los días, un $21.6 \%$ algunas veces por semana, mientras que el $6.6 \%$ nunca desayunaba. Al agrupar los jóvenes según condición de omitir el desayuno, se observó que un $71.8 \%$ no lo omitía, es decir tenía el hábito de desayunar, mientras que el $28.2 \%$ restante omitía este tiempo de comida.

Al estudiar la existencia de asociación entre la omisión del desayuno y las variables sexo, edad, estado nutricional, información sobre el desayuno y condición de estar solo en este tiempo de comida, se encontraron los siguientes resultados:

En lo relativo al sexo de los adolescentes, se observó que, entre quienes omitían desayunar un $51.3 \%$ eran del sexo femenino y un $48.7 \%$ eran de sexo masculino. No se encontró asociación estadísticamente significativa entre la omisión del desayuno y el sexo $(p=0.203)$ (Tabla 1$)$.

En relación a la edad, entre quienes realizaban su desayuno diariamente, un $50.7 \%$ tenían más de 13 años y aquellos que lo omitían un $51.5 \%$ tenían esta edad siendo prácticamente la misma proporción. No se encontró asociación entre la omisión del desayuno y la edad de los jóvenes ( $p=0.844$ ) (Tabla 1$)$.

Entre quienes desayunaban, el $35.7 \%$ si presentaba algún grado de exceso de peso, mientras que un $64.3 \%$ no presentaba ni sobrepeso ni obesidad. En relación a los que omitían el desayuno, el 40,4\% presenta algún grado de sobrepeso u obesidad, mientras que casi el $60 \%$ (59.6\%), no presentaba esta condición. Por lo que no existe asociación estadísticamente significativa entre la omisión del desayuno y el estado nutricional de los adolescentes $(p=0.254)$ (Tabla 1$)$.

Entre los jóvenes que omitían el desayuno un 78.1\% fue informado de alguna manera, sobre la importancia de este tiempo de comida. Por otra parte, un $\mathbf{7 5 . 4 \%}$ de los que desayunaban también mencionaron recibirla. La diferencia es prácticamente nula entre los dos grupos, no presentándose asociación estadísticamente significativa entre ambas variables $(p=0.460)(T a-$ bla 1).

Por último, al estudiar la condición de estar solo en este tiempo de comida se obtuvo que, del total de estudiantes, un $43.9 \%$ lo hacía solo, un $54.9 \%$ con familiares y un $1.2 \%$ con compañeros. Entre aquellos adolescentes que desayunaban, un 39.3\% se encontraba solo, y en aquellos que omitían desayunar diariamente, la frecuencia de desayuno sin compañía fue de un $59.3 \%$ (Gráfico 1). Al estudiar la asociación entre la condición de estar solo y la omisión del desayuno, se encontró asociación estadísticamente significativa $(p=0.00)$. Así mismo, la probabilidad de omitir el desayuno con respecto a no omitirlo es aproximadamente 2,25 más alto en los adolescentes que se encuentran solos con respecto a los que se encuentran acompañados (OR: 2.256; 95\% IC. 1.555- 3.272) (Tabla 1). 
Al estudiar los adolescentes que omitían el desayuno $(n=196)$, se observó que $51.3 \%$ eran mujeres, y $48.7 \%$ eran varones. Las edades se encontraban entre los 11 y 17 años, siendo la media 13.2 años y la mediana 14 años.

Si se toma la media de edad en 13 años, un $48.5 \%$ de los jóvenes tenían al momento de la encuesta 13 años o menos, mientras que un $51.5 \%$ eran mayores de 13 años. Se observó una distribución similar por sexo y edad.

Según grado escolar al que pertenecían, el 29.1\% era de primer año de secundaria, el $26.5 \%$ de segundo año, el $21.9 \%$ de tercer año y el $22.5 \%$ de cuarto año o primer año de bachillerato. Un $61.7 \%$ de la población estudiada asistía al centro educativo en el horario de la mañana y un 32,1\% lo hacía en ambos turnos. El horario promedio de levantarse los días que acudían al colegio fue las $7 \mathrm{am}$.

Un 59,6\% de los adolescentes presentó un estado nutricional normal según IMC/E, un 29,2\% presentó sobrepeso y un $11,2 \%$ obesidad. Para el caso específico de los jóvenes que omiten el desayuno, el 40,4\% presentaba sobrepeso u obesidad. Un 1.6\% del total de adolescentes presentó talla baja para la edad. Para el resto, su talla era normal para su edad.

Se observó que entre los adolescentes que tenían 13 años o menos un $48.4 \%$ presentaba un estado nutricional normal, mientras que más de la mitad de ellos, el $51.6 \%$ presentaba algún grado de sobrepeso u obesidad. Casi un tercio de los jóvenes que omitieron el desayuno y eran mayores de 13 años de edad presentaron sobrepeso u obesidad (29.9\%), existiendo asociación estadísticamente significativa entre la edad de los adolescentes que omitían el desayuno y el estado nutricional $(p=0.002)$.

De todos los adolescentes que relataron omitir el desayuno, un $78.1 \%$ mencionó haber recibido algún tipo de información sobre la importancia de realizarlo. Al analizar la fuente de información referida, se observó que el ámbito médico y el colegio son los dos sitios mencionados con más frecuencia, con un $28.3 \%$ y $25.3 \%$ respectivamente, seguido de la casa y la televisión como sitios de información.

Al analizar los diferentes motivos referidos por los adolescentes para explicar la omisión del desayuno, se observa en el Gráfico 2 que el hecho de "levantarse tarde y no tener tiempo" fue la razón más mencionada (37.8\%) seguida por la "falta de apetito" (32.4\%). Se analizó el primer motivo mencionado con más frecuencia según la edad, el sexo y el estado nutricional.

Se encontró asociación estadísticamente significativa entre la edad y la razón "levantarse tarde y no tener tiempo" ( $p=0.017)$. Los jóvenes mayores de 13 años relataron este motivo con más frecuencia (60.2\%) que los de 13 años de edad o menos (39.8\%) (Tabla 2).

Por otra parte, los varones refirieron este motivo en mayor porcentaje (56.7\%) que las mujeres (43.3\%). Existe asociación estadística entre "levantarse tarde y no tener tiempo" y el sexo $(p=0.035)$ (Tabla 2).
Fue mayor el porcentaje de adolescentes con estado nutricional normal (61.9\%) que refirió el motivo de "no tener tiempo para desayunar", comparados con los que presentaron algún grado de sobrepeso u obesidad y expresaron este motivo como el principal (38.1\%). No se encontró asociación entre el estado nutricional y este motivo para no desayunar $(p=0.537)$ (Tabla 2).

\section{DISCUSIÓN}

Los resultados del presente estudio permiten conocer algunas características relacionadas a la omisión del desayuno por parte de los adolescentes de 11 a 17 años que asisten a colegios privados de la ciudad de Montevideo.

Más de un cuarto de los jóvenes omite la primer comida del día o desayuno (28.2\%). Esta cifra es similar a la encontrada en estudios de Estados Unidos y Europa, en los cuales entre $10 \%$ a $30 \%$ de los adolescentes no desayunan de manera habitual (4). Las recomendaciones de la Asociación Española de Pediatría y de las Guías alimentarias de nuestro país hacen referencia a la importancia de un desayuno diario (3) (14).

Si bien algunas investigaciones sobre el tema han descripto asociación entre el sexo de los adolescentes y el saltearse el desayuno, en este estudio no se pudo demostrar la asociación estadística entre estas variables. Según la bibliografía, es más frecuente la omisión de este tiempo de comida en las jóvenes, puesto que, entre otros motivos, suelen estar más preocupadas por su peso corporal, llevándolas a pasar largos períodos de ayuno como estrategia para el control calórico (11) (6) (7) (10). Este último factor no ha sido indagado en esta investigación, pero es de considerar esta característica comportamental para establecer los mensajes educativos adecuadamente.

Resultados obtenidos en el estudio AVENA en España, entre otros, (11) (6) (15) (10), hacen referencia a la asociación entre la edad de los adolescentes y la condición de omitir el desayuno. A medida que avanza la edad el hábito de desayunar desciende (7). Los resultados obtenidos en este trabajo no muestran asociación entre estas variables, sólo se logra ver una diferencia de 3 puntos porcentuales entre los mayores de 13 años que omiten el desayuno y los menores.

El estado nutricional podría haber sido un factor que se asociara a la omisión del desayuno en esta población. Varios estudios han demostrado que a mayor peso corporal, se observa un aumento en la prevalencia de la supresión de este tiempo de comida (6) (16) (15). En este estudio, sin embargo, se halló que, entre quienes no omiten desayunar un $35.7 \%$ presentaba sobrepeso u obesidad, mientras que entre los que saltean este tiempo de comida la cifra asciende a un $40.4 \%$, no siendo estas diferencias estadísticamente significativas. Esto se puede deber a que existen muchas variables que inciden en el estado nutricional de los adolescentes, especialmente en relación al desarrollo de sobrepeso y obesidad. Además de la influencia del hábito de desayunar, está la selección de alimentos en cantidad y calidad, la ingesta diaria de energía y nutrientes, el tipo 
y frecuencia de actividad física, el sedentarismo relacionado a las pantallas, las horas destinadas al descanso y al sueño, los antecedentes personales y familiares, entre otras.

Según OMS, existe un mayor riesgo de padecer sobrepeso u obesidad, de forma convincente, si se tiene un estilo de vida sedentario, y si existe una elevada ingesta de alimentos ricos en energía y bajos en micronutrientes. Como relación probable menciona la publicidad masiva de alimentos ricos en energía y lugares de comida rápida, una ingesta elevada de refrescos y jugos azucarados, y condiciones socioeconómicas adversas (17). Para poder afirmar la relación entre el estado nutricional y la omisión del desayuno, hubiese sido necesario indagar los demás aspectos que hacen al estado nutricional de sobrepeso y obesidad y de esta manera poder controlarlos y medirlos adecuadamente.

Casi un $80 \%$ de los jóvenes que omiten desayunar han sido informados sobre la importancia de realizar este tiempo de comida. Si bien no se encontró asociación entre estas variables, las cifras muestran que, en esta población, solo el hecho de brindar información sobre esta temática no es condición suficiente para alcanzar el hábito de desayunar cotidianamente.

Al analizar la omisión del desayuno junto a la condición de estar solo en este tiempo de comida, se obtiene que existe una asociación estadísticamente significativa entre ambas variables $(p=0.00)$. A su vez, existen mayores chances de omitir el desayuno, si el adolescente se encuentra solo. Resultados similares se obtuvieron en otros estudios (11), siendo éste un dato de suma relevancia y que debemos resaltar a la hora de realizar consejería alimentaria y nutricional individual, familiar o colectiva. En el estudio de Amat Huerta y cols. se vio que el número de jóvenes que desayuna en familia es inferior al que lo hace sólo (12). Las comidas en familia constituyen una actividad central y un particular punto de encuentro en la vida diaria de sus miembros, fortaleciendo la unidad y cohesión familiar. Es muy útil usar el desayuno en familia como forma de educar los hábitos alimentarios de los jóvenes. No se estudiaron las barreras que pueden existir para desayunar en familia, sin embargo, a la vista de los resultados obtenidos, sería importante conocerlas en profundidad para poder trabajar sobre ellas. Diversos estudios relatan que la falta de tiempo y la diferencia de horarios entre los padres e hijos podrían ser la explicación (12). La OMS en su informe Dieta, Nutrición y prevención de Enfermedades Crónicas recomienda crear más oportunidades para las relaciones familiares incentivando las comidas en familia (17) por otro lado, diversas Guías alimentarias de otros países han incluido dentro de sus mensajes a la población, la importancia de comer acompañado (18) (19).

En relación a las características que presenta la población de adolescentes que omite el desayuno, ésta se divide proporcionalmente según la edad y el sexo. La distribución de adolescentes según grado escolar fue similar. Con respecto al turno de asistencia al centro educativo se observa que un $93.8 \%$ de los jóvenes lo hace desde la mañana temprano, siendo la hora promedio de levantarse las 7 am. Por lo tanto, el horario y las rutinas de los adolescentes que concurren a colegios privados puede ser un variable a atender en el estudio del hábito de desayunar de los jóvenes y de esta manera, realizar las estrategias para la promoción de este tiempo de comida de forma diaria y sistemática.

Al estudiar el estado nutricional actual de los adolescentes que omiten el desayuno, la prevalencia de sobrepeso- obesidad fue del $40.4 \%$, presentándose la obesidad en un $11.2 \%$. Por lo tanto, 4 de cada 10 adolescentes estudiados tienen un IMC para su edad mayor al esperado según los estándares de referencia. El porcentaje de estudiantes con obesidad es casi 5 veces mayor al esperado para esta población (2.3\%). Este valor es superior al encontrado en el estudio ENSO I (20) y a los resultados obtenidos en la II Encuesta Mundial de Salud Adolescente (11).

El porcentaje de estudiantes con talla baja para la edad no representa un problema, siendo éste menor al esperado para esta población (2.3\%).

Casi el $80 \%$ de los adolescentes que omiten desayunar relatan haber recibido información sobre el desayuno en algún momento de sus vidas, siendo el médico una de las fuente de información más mencionada. Parece importante replantear las estrategias para fomentar el desayuno desde el ámbito de la salud. Conocer en profundidad las necesidades, barreras y motivaciones de este grupo etario resulta fundamental, para de esta manera brindar mensajes acordes a los adolescentes, que logren un impacto en el cambio de conducta. El modelo de "Atención Integral del adolescente" del MSP, busca entre otras cosas, la participación activa de los jóvenes en su salud (21). Esta etapa de la vida se caracteriza por la búsqueda de la propia identidad y por la independencia recién adquirida. Si logramos empoderarlos en su proceso de cambio y hacerlos protagonistas, probablemente obtengamos mejores resultados.

El estudio de los motivos por los cuales los jóvenes suelen no realizar el desayuno a diario, es de gran relevancia para poder comprender mejor dicho comportamiento.

El "levantarse tarde y no tener tiempo", es la razón más frecuente entre quienes omiten realizarlo, esto se podría explicar porque el mayor porcentaje de adolescentes asiste al centro educativo en el turno matutino y por otro lado, si bien no se indagó, generalmente suelen acostarse tarde de noche entretenidos con la televisión, computadora y juego electrónicos, quitándoles esto horas de sueño. Acostarse tarde y levantarse con poco tiempo termina siendo un círculo vicioso que repercute en la omisión del desayuno.

Cuando se indaga en el motivo "levantarse tarde y no tener tiempo" y su relación con la edad se observa que a mayor edad éste es más frecuente, un $60.2 \%$ de los que lo relatan como una razón para no desayunar son mayores de 13 años, existiendo una asociación estadísticamente significativa entre estas variables. Probablemente se deba a la razón anteriormente mencionada, a mayor edad mayor independencia y una búsqueda de menor control parental influyendo esto en las horas de descanso de los jóvenes. También resulta haber una asociación significativa entre este motivo y el sexo. Los varones argumentan con más frecuencia que tienen menos tiempo que las chicas a esa hora de la mañana. El estado nutricional, en esta población, no parece influenciar en la falta de tiempo para desayunar. 
Es necesario tener en cuenta las potenciales limitaciones de este estudio, que se deben a aspectos relacionados con la forma de interrogar la frecuencia con que los adolescentes realizaban el desayuno. Las opciones que podían indicar en el formulario auto-administrado eran: Siempre, Algunas veces o Nunca. No se conoce la frecuencia exacta para Algunas veces a la semana, pudiendo ser muy variable. Esto se debe a que los datos no fueron recabados para realizar una investigación sobre la omisión del desayuno, sino que provienen de una fuente secundaria de información, con fines descriptivos.

\section{CONCLUSIONES}

Pese a los reconocidos beneficios de realizar el desayuno, se constata que más de un cuarto de los adolescentes omite desayunar a diario.

Si bien las chicas omiten desayunar en mayor porcentaje (51.3\%) que los varones (48.7\%), no se pudo establecer la asociación entre estas variables. Tampoco se asoció la edad y el estado nutricional con la omisión de este tiempo de comida.

No se encontró asociación estadísticamente significativa entre el recibir información y la omisión del desayuno, sin embargo se observó que, un elevado porcentaje de adolescentes que omiten desayunar han sido informados sobre la importancia de esta temática, especialmente por el médico, por lo que se puede concluir que el sólo hecho de brindar información no parece ser suficiente para fomentar este hábito, en esta población, y lograr cambios de comportamiento que favorezcan el cuidado en salud.
Existen mayores chances de omitir el desayuno si el adolescente se encuentra solo en este tiempo de comida. Este resultado pone de manifiesto la importancia de la familia en la instauración de hábitos dietéticos. Incluir a los padres o adultos responsables de la crianza como agentes de cambio parece ser una de las claves a considerar. Se confirma la importancia del rol de la familia en la instauración de hábitos de desayuno saludables en estos adolescentes.

Al igual que en otros estudios llevados a cabo en población adolescente, el sobrepeso y la obesidad es un problema de gran magnitud en los jóvenes que omiten desayunar, siendo mayor la proporción en aquellos adolescentes de 13 años o menos.

El "levantarse tarde y no tener tiempo" resultó el motivo más mencionado para no realizar habitualmente este tiempo de comida. Siendo los varones y los adolescentes mayores de 13 años quienes más mencionan este argumento.

Se recomienda considerar los resultados y conclusiones mencionados anteriormente para trazar líneas de acción dirigidas especialmente a este grupo etario. Pensar estrategias en el marco de las políticas públicas en nutrición, con enfoque sobre los adolescentes que fomenten el destinar un tiempo diario para desayunar.

Parece de suma relevancia empoderar a la familia en el acto de comer juntos, revalorizando su papel en el desarrollo de las rutinas alimentarias incluido el hábito de desayunar. Esta etapa de la vida parece ser una segunda ventana de oportunidad para actuar, reconociendo que los adolescentes de hoy son la base de la sociedad del mañana. Debemos apoyar la transición hacia la edad adulta, utilizando un enfoque de abordaje integral. 
Tabla 1: Sexo, edad, estado nutricional, información recibida y condición de estar solo al desayunar según omisión del desayuno

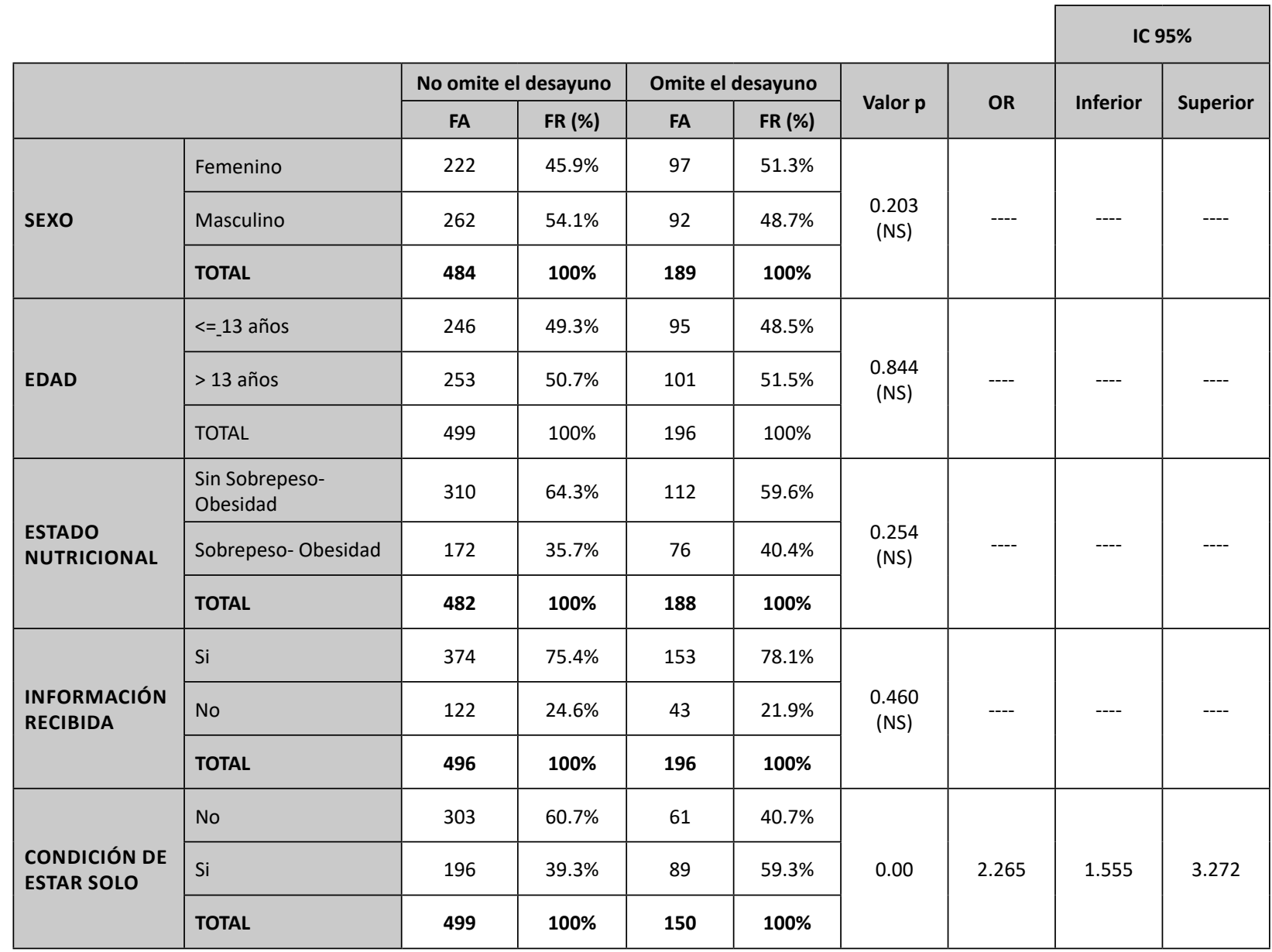

\section{$70.0 \%$ \\ $60.0 \%$

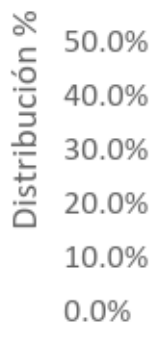

$60.7 \%$

$40.7 \%$

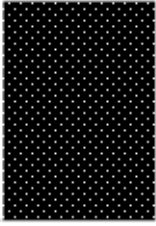

Acompañado

No omite desayuno
$59.3 \%$

$39.3 \%$

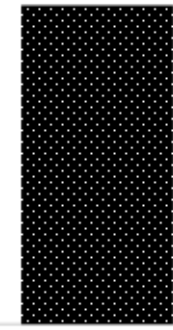

Solo 


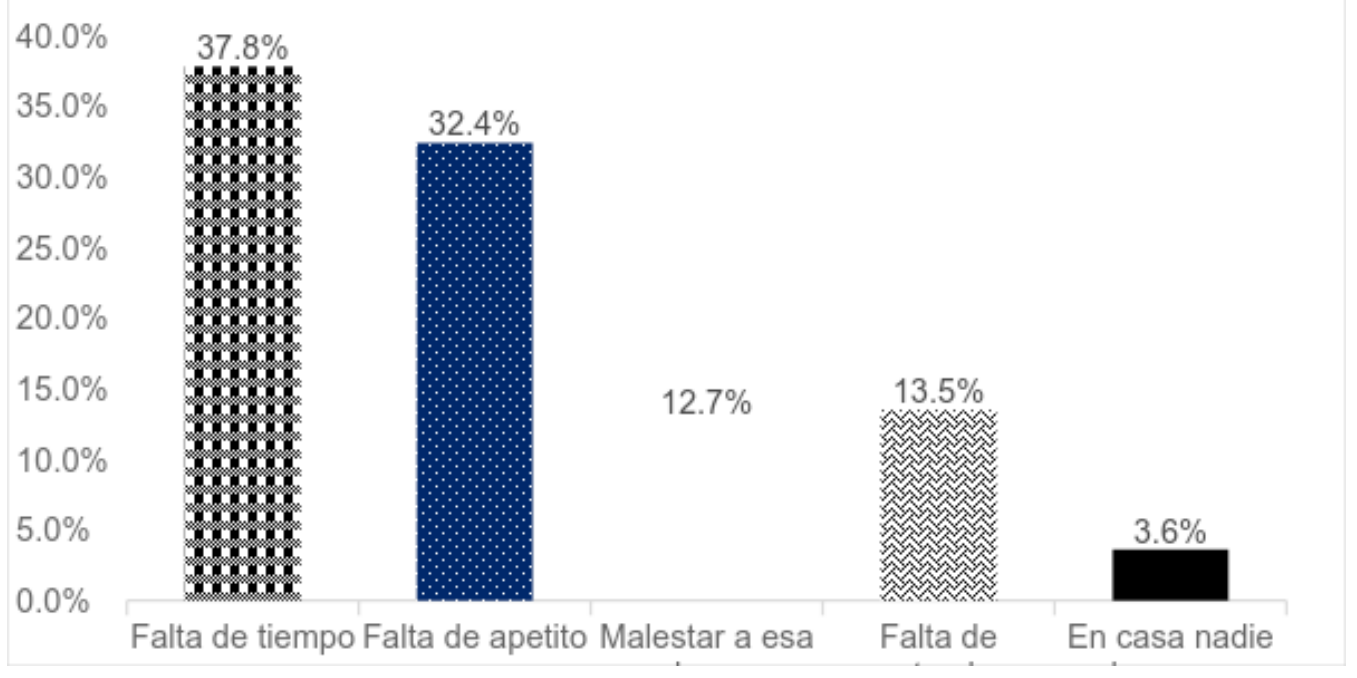

Gráfico 2: Distribución porcentual de los motivos mencionados para omitir el desayuno

Tabla 2: Motivo "Levantarse tarde y no tener tiempo" según sexo, edad y estado nutricional de los adolescentes

\begin{tabular}{|c|c|c|c|c|c|c|}
\hline & & \multicolumn{5}{|c|}{ FALTA DE TIEMPO } \\
\hline & & \multicolumn{2}{|c|}{$\mathrm{SI}$} & \multicolumn{2}{|c|}{ No } & \multirow{2}{*}{ Valor $\mathrm{p}$} \\
\hline & & FA & FR (\%) & FA & FR (\%) & \\
\hline \multirow{3}{*}{ SEXO } & Femenino & 42 & $43.3 \%$ & 49 & $59 \%$ & \multirow{3}{*}{0.035} \\
\hline & Masculino & 55 & $56.7 \%$ & 34 & $41 \%$ & \\
\hline & TOTAL & 97 & $100 \%$ & 83 & $100 \%$ & \\
\hline \multirow{3}{*}{ EDAD } & $<=13$ años & 39 & $39.8 \%$ & 51 & $57.3 \%$ & \multirow{3}{*}{0.017} \\
\hline & $>13$ años & 59 & $60.2 \%$ & 38 & $42.7 \%$ & \\
\hline & TOTAL & 98 & $100 \%$ & 89 & $100 \%$ & \\
\hline \multirow{3}{*}{$\begin{array}{l}\text { ESTADO } \\
\text { NUTRICIONAL }\end{array}$} & Normopeso & 60 & $61.9 \%$ & 47 & $57.3 \%$ & \multirow{3}{*}{0.537 (NS } \\
\hline & Sobrepeso- Obesidad & 37 & $38.1 \%$ & 35 & $42.7 \%$ & \\
\hline & TOTAL & 97 & $100 \%$ & 82 & $100 \%$ & \\
\hline
\end{tabular}


Cómo citar este artículo: Ceriani Infantozzi, F. De León-Giordano, C. Características que se asocian con la omisión del desayuno en adolescentes montevideanos que concurren a colegios privados. Enfermeria (montev.). [Internet] 2017 Dic. [citado xxxxx]; 6 (2) 12-20. Disponible en: https://doi. org/10.22235/ech.v6i2.1461

\section{REFERENCIAS BIBLIOGRÁFICAS}

1. Moreno Aznar L, Rodríguez Martínez G. Nutrición en la adolescencia. In Gil Hernández Á. Tratado de Nutrición.: Médica Panamericana; 2010. p. 371389.

2. Castañeda Sánchez O, Rocha Díaz J, Ramos Aispuro M. Evaluación de los hábitos alimenticios y estado nutricional en adolescentes de Sonora, México. Archivos en Medicina Familiar. 2008; 10 (1) 7- 9.

3. Dalmau Serra J, Alonso Franch M, Gómez López L, Martínez Costa C, Sierra Salinas C. Obesidad Infantil. Recomendaciones del Comité de Nutrición de la Asociación Española de Pediatría. Parte II. Diagnóstico. Comorbilidades. Tratamiento. An Pediatr (Barc). 2007; 66(3).

4. Affinita A, Catalani L, Zuccotti G. Breakfast a multidisciplinary approach. Ital J Pediatr. 2013; 39(44).

5. Mullan B, Wong C, Kothe E, O'Moore K, Pickles K, Sainsbury K. An examination of the demographic predictors of adolescent breakfast consumption, content, and context. BMC Public Health. 2014; 14(264).

6. Rampersaud G, Pereira M, Girard B, Adams J, Metzl J. Breakfast Habits, Nutritional Status, Body Weight, and Academic Performance in Children and Adolescents. J Am Diet Assoc. 2005; 105(743-760).

7. Wärnberg J, Ruiz JR, Ortega FB, Romeo J, González Gross M, Moreno LA, et al. Estudio AVENA (alimentación y valoración del estado nutricional en adolescentes). Pediatr Integral; 2006 p. 50-55.

8. De León C, Pereyra I. Estudio de hábitos alimentarios en el desayuno de adolesccentes de liceos privados de Montevideo

9. Díaz Fernández R. Adolescentes y alimentación: factores que inciden en los comportamientos alimentarios. In I Congreso Español de Sociología de la Alimentación, Gijón (La Laboral); 2009.
10. Kapantais E, Chala E, Kaklamanou D, Lanaras L, Kaklamanou M, Tzotzas T. Breakfast skipping and its relation to BMI and health-compromising behaviours among Greek adolescents. Public Health Nutrition

11. GSHS. Adolescencias: un mundo de preguntas II Encuesta Mundial de Salud Adolescente. Uruguay: Organización Panamericana de la Salud, Junta Nacional de Drogas, Ministerio de Salud Pública, Ministerio de Desarrollo Social; 2012.

12. Amat Huerta M, Anuncibay Sánchez V, Soto Volante J, Alonso Nicolás N, Villalmanzo Francisco A, Lopera Ramírez S. Estudio descriptivo sobre hábitos alimentarios en el desayuno y almuerzo de los preadolescentes de Viladecans (Barcelona). Nure investigación;. 2006 Julio

13. OMS. Elaboración de un patrón OMS de crecimiento de escolares y adolescentes. [Online]

14. Manual para la promoción de prácticas saludables de alimentación en la población uruguaya. 2007. Ministerio de Salud Pública, Programa Nacional de Nutrición.

15. Quintero-Gutiérrez A, González G, Rodríguez N, Reyes G, Puga R, Villanueva J. Omisión del desayuno, estado nutricional y hábitos alimentarios de niños y adolescentes de escuelas públicas de Morelos, México. CyTA - Journal of Food. 2014 Febrero; 12:3.

16. Currie C, Zanotti C, Morgan A, Currie D, de Looze M, Roberts $C$, et al., editors. Social determinants of health and well-being among young people. Health Behaviour in School-aged Children (HBSC) study: Copenhagen: WHO Regional Office for Europe, 2012 (Health Policy for Children and Adolescents, No. 6); 2012.

17. OMS sdit. Programación para la salud y el desarrollo de los adolescentes. Informe de un grupo de estudio OMS/ FNUAP/UNICEF sobre la programación para la salud de los adolescentes. Ginebra; 1999.

18. Guía alimentaria para la población brasileñ

19. Silva L, Longo E, Lopresti A. Guias alimentarias: manual de multiplicadoras

20. Pisabarro R, Irrazabal E, Recalde A, Chaftare Y. ENSO niños 1: Primera encuesta nacional de sobrepeso y obesidad en niños uruguayos. Revista Médica del Uruguay

21. Guías para el abordaje integral de la salud de adolescentes en el primer nivel de atención. Tomo 1. Uruguay: Ministerio de Salud Pública, Dirección general de la Salud, Programa Nacional de Salud Adolescente; 2009. 